\title{
Capturing cooperativity
}

\author{
Considering common definitions and mechanisms of cooperative behavior may lead to new insights into chemical and \\ biological function.
}

C ooperativity is well known as the energetic basis for a variety of microscopic events, such as the simultaneous chelation of metal ions, the temporal coordination of protein folding and the concerted function of biomolecular assemblies. At the macroscale, cooperation between scientific laboratories, organizations and countries is required to advance research in a timely manner and to coordinate conferences and funding initiatives. Although the species under consideration and the consequences of the cooperative behavior in these cases are not clearly interrelated, the underlying mechanisms of these activities may share similar features. Accordingly, we suggest that as our detailed understanding of complex biological processes increases, consideration of what cooperativity means at different scales as well as consideration of the commonalities of these processes will further advance our understanding of intricate systems.

It is not surprising that the term 'cooperativity' is most clearly tied to small-molecule properties and enzyme behavior. The cooperative function of hemoglobin was first documented in 1925 by Gilbert Adair, although the conceptual framework to describe these data took additional time to develop (as revisited in J. Biol. Chem. 277, e20, 2002). Similarly, the term 'chelate' was first coined in 1920 (J. Chem. Soc. 117, 1456-1465, 1920), with subsequent investigations into host molecules such as clathrates and cryptands cementing these compounds in the modern understanding of cooperative interactions.

But what does 'cooperativity' mean today? Cooperativity encompasses a range of scientific systems and provides an umbrella term for processes such as preorganization, avidity, allostery and some types of assembly. Cooperativity can also be more broadly defined as a process for which intermediates are disfavored (resulting, for example, in a two-state conformational change). Our increasing ability to discern the individual steps of protein folding or to detect subtle structural changes induced by biomolecular interactions, however, complicates this analysis: with more information at hand, it becomes less clear that a particular process is cooperative as compared to simply proceeding along a downward energetic trajectory or occurring at an observed rate. Similarly, according to this broad definition, complicated processes such as cytokinesis can be considered cooperative in that in the absence of perturbants or disruptive mutations, halting at an intermediate state is disfavored. As our mechanistic insight into biological systems grows, scientists must be mindful not to apply the term too loosely to processes that are simply coordinated in space or time (like cell division or signaling cascades), but rather they must look for those systems that are directly energetically linked.

Chemical biologists will be important contributors in this ongoing research. As scientists who are both committed to understanding chemical and biological problems at the most detailed level and well trained in the fundamentals of thermodynamics and kinetics, chemical biologists are poised to evaluate how and when systems are cooperative. This analysis, while somewhat simplified at the level of individual molecules, becomes complicated as additional pieces come into play. The development of new techniques to allow more thorough investigations (Commentary, p. 440) and new tools to probe complex machinery (Nat. Chem. Biol. 4, 3-6, 2008) will be critical in quantifying biomolecular motions and function. Application of these tools will both expedite our evaluation of cooperative systems and provide access to greater molecular detail.

Research on cooperative systems has already benefited from these advances as well as from the application of the more mechanistic chemical biology perspective more generally. The initial studies on hemoglobin have blossomed, in some cases, into a detailed knowledge of specific amino acid networks that communicate to confer allosteric conformational changes in binding and catalysis (Reviews, p. 474). Experimental limitations on the sizes or numbers of individual biomolecules that can be studied have concurrently decreased, with the result that macromolecular assemblies are now beginning to be quantified in a meaningful way (Perspective, p. 458). This progress suggests that systems like the nuclear pore complex (Nature 450,695-701, 2007), once thought to be intractable, will eventually yield their secrets. Increasing analytical capabilities and genomic information are providing new information about how cooperativity can occur at the cellular and organismal levels in symbiotic species (Reviews, p. 466) and in social insects such as the honeybee (Nature 443, 931-949, 2006). Even human interactions are generating new insights and accolades: the subtleties of human nature in cooperating with colleagues (Nature 451, 189-192, 2008; Nature 452, 348-351, 2008) may be trumped only by the confusion that can arise in regulating such interactions (Nature 452, 682-684, 2008). Even with the myriad of complexities governing interpersonal relations, we do see examples of cooperation in science, both on local (Chem. Eng. News 86, 50-51, 2008) and global scales (see, for example, the American Association for the Advancement of Science Award for International Scientific Cooperation, http://www.aaas.org/aboutaaas/awards/int/int_winners.shtml).

These diverse examples accentuate the importance of looking at cooperativity from new angles, in allowing us to formulate hypotheses about increasingly complex systems and in helping to provide a firm scientific grounding for discussions about larger scale phenomena such as emergent properties (Commentary, p. 435). Chemical biology itself provides an important reflection of cooperativity, since the field has grown through collaboration and openness to new ideas and approaches. We hope this issue, which features pieces exploring molecular, cellular and organismal cooperativity, inspires further thought as to how the mechanisms of seemingly divergent systems intersect. 